\title{
MULTIPLE EXPANSIONS IN SERIES OF BOAS AND BUCK POLYNOMIALS
}

\author{
BY RICHARD F. DE MAR1
}

Communicated by W. T. Martin, December 10, 1971

In Boas and Buck's Ergebnisse monograph [1] they deal with expansions of analytic functions in series $f(z)=\sum a_{n} p_{n}(z)$ where the sequence $\left\{p_{n}\right\}$ of polynomials satisfies a formal generating relation

$$
A(w) \Psi(z g(w))=\sum_{n=0}^{\infty} p_{n}(z) w^{n}
$$

with $A(0) \neq 0, g(0)=0, g^{\prime}(0) \neq 0, A$ and $g$ analytic on a simply connected domain $\Omega$ containing the origin, and $\Psi(t)=\sum \psi_{n} t^{n}$ where $\psi_{n}>0$ and $\psi_{n+1} / \psi_{n} \downarrow 0$. They call these generalized Appell polynomials, but others have used this name for other sequences of polynomials and the above have become known as Boas and Buck polynomials (e.g. [2], [3]).

In the monograph, they illuminate some of the causes of multiple expansions of functions in such series; i.e.

$$
f(z)=\sum_{n=0}^{\infty} a_{n} p_{n}(z)=\sum_{n=0}^{\infty} b_{n} p_{n}(z)
$$

without having $a_{n}=b_{n}$ for all $n$, or equivalently, nontrivial representations of zero;

$$
\sum_{n=0}^{\infty} h_{n} p_{n}(z) \equiv 0
$$

without all $h_{n}=0$. For example, if $A\left(w_{0}\right)=0$, we have

$$
\sum_{n=0}^{\infty} w_{0}^{n} p_{n}(z) \equiv 0
$$

If $w_{0}$ is a double zero of $A$, then differentiation also gives

$$
\sum_{n=1}^{\infty} n w_{0}^{n-1} p_{n}(z) \equiv 0
$$

and similarly for higher order zeros of $A$.

Also, nontrivial representations of zero may arise from the nonuni-

AMS 1970 subject classifications. Primary 30A62.

Key words and phrases. Multiple expansions, Boas and Buck polynomials, nontrivial representations of zero, Mittag-Leffler summable.

1 This work was supported in part by National Science Foundation Grant \#9619. 
valence of $g$; say $g\left(w_{1}\right)=g\left(w_{2}\right)$ for $w_{1} \neq w_{2}$ in $\Omega$ where $A\left(w_{1}\right) \neq 0$, $A\left(w_{2}\right) \neq 0$. Then

$$
\sum_{n=0}^{\infty}\left[\frac{w_{1}^{n}}{A\left(w_{1}\right)}-\frac{w_{2}^{n}}{A\left(w_{2}\right)}\right] p_{n}(z) \equiv 0 .
$$

The question can then be asked: Are these the only sources of nontrivial representations of zero? Boas and Buck give a partial answer with the theorem [1, p. 26]:

Let $g$ be univalent on $\Omega$ and let

$$
\sum_{n=0}^{\infty} h_{n} p_{n}(z) \equiv 0
$$

uniformly convergent on all compact subsets of the plane where $h_{n}=O\left(R^{n}\right)$ for some finite $R$ such that the disk $|w| \leqq R$ is a subset of $\Omega$. Then the representation (2) of zero arises from zeros of $A(w)$ in $\Omega$.

The conditions put on the $h_{n}$ in the theorem are precisely those that they have used earlier to guarantee convergence of (2). Thus, this theorem deals with all representations of zero in which the given theory implies convergence of the series. But they also deal with representations of functions by Mittag-Leffler summable series. A series $\sum_{n=0}^{\infty} a_{n}$ is MittagLeffler summable to $A$ if $\lim _{\delta \rightarrow 0} \sum_{n=0}^{\infty}\left(a_{n} / \Gamma(1+\delta n)\right)$ exists and equals $A$. It is a totally regular method of summation and has the property that if $f(z)=\sum_{n=0}^{\infty} c_{n} z^{n}$ is analytic on a star domain $D$ (i.e. $z \in D$ implies $\lambda z \in D$ for all $\lambda$ such that $0 \leqq \lambda \leqq 1)$ then $\sum c_{n} z^{n}$ is Mittag-Leffler summable to $f$ on $D$, uniformly on compact subsets of $D$. We denote the Mittag-Leffler sum of a series by ML- $\sum_{n=0}^{\infty} a_{n}$ and write ML-summable for MittagLeffler summable. In many applications of the theory developed by Boas and Buck, ML-summability gives a much larger class of representable functions than does convergence.

Again, multiple expansions may arise from zeros of $A(w)$ and from nonunivalence of $g(w)$ and again one can ask if these are the only ways they can arise. The reason that convergence was needed in the proof given in Boas and Buck is that it involved the multiplication of power series and the fact that the product converges wherever both factors converge. With summability, one does not have this property. We show here that a careful ordering of operations eliminates the necessity of multiplying series and yields the expected analogous result for ML-summable series.

THEOREM. Let $\left\{p_{n}\right\}$ be a sequence of Boas and Buck polynomials; i.e. satisfy a generating relation (1), and let $\Omega$ be a star domain with $g$ univalent on $\Omega$. Let

$$
M L-\sum_{n=0}^{\infty} h_{n} p_{n}(z) \equiv 0
$$


for all $z$, uniformly on compact subsets of the plane; where the sequence $\left\{h_{n}\right\}$ is such that not all $h_{n}=0$ and

$$
H(w)=M L-\sum_{n=0}^{\infty} \frac{h_{n}}{w^{n+1}}
$$

is analytic on $\Omega^{c}$. Then (3) arises from zeros of $A(w)$ in $\Omega$.

REMARK. The condition on $\left\{h_{n}\right\}$ is the exact analogue for ML-summability of that used by Boas and Buck for convergence.

Proof. Since $H(w)$ is analytic on $\Omega^{c}$ and $\Omega^{c}$ is a closed set, starlike with respect to $\infty, \sum_{n=0}^{\infty} h_{n} w^{-n-1}$ is uniformly ML-summable on compact subsets of a domain containing $\Omega^{c}$. Let $\Gamma$ be a simple closed contour contained in $\Omega$, enclosing a star domain, and such that $H$ is analytic outside and on $\Gamma$. Define

$$
K(z)=\frac{1}{2 \pi i} \int_{\Gamma} A(w) \Psi(z g(w)) H(w) d w .
$$

Since for each $z, A(w) \Psi\left(z g\left(w^{\prime}\right)\right)$ is analytic in $w$ inside and on $\Gamma, \sum p_{n}(z) w^{n}$ is uniformly ML-summable on $\Gamma$, and we have

$$
\begin{aligned}
K(z) & =\mathrm{ML}-\sum_{n=0}^{\infty} p_{n}(z) \frac{1}{2 \pi i} \int_{\Gamma} w^{n} H(w) d w \\
& =\mathrm{ML}-\sum_{n=0}^{\infty} p_{n}(z) \frac{1}{2 \pi i} \int_{\Gamma} w^{n} \mathrm{ML}-\sum_{k=0}^{\infty} \frac{h_{k}}{w^{k+1}} d w \\
& =\mathrm{ML}-\sum_{n=0}^{\infty} p_{n}(z)\left(\mathrm{ML}-\sum_{k=0}^{\infty} h_{k} \frac{1}{2 \pi i} \int_{\Gamma} \frac{d w}{w^{k-n+1}}\right) \\
& =\mathrm{ML}-\sum_{n=0}^{\infty} p_{n}(z) h_{n} \\
& \equiv 0 .
\end{aligned}
$$

From this point on, the proof given in Boas and Buck [1, pp. 26-27] goes through without modification and gives the conclusion of our theorem.

\section{REFERENCES}

1. R. P. Boas, Jr. and R. C. Buck, Polynomial expansions of analytic functions, rev. ed., Ergebnisse der Mathematik und ihrer Grenzgebiete, N.F., Band 19, Academic Press, New York; Springer-Verlag, Berlin, 1964. MR 29 \#218.

2. J. L. Goldberg, A note on polynomials generated by $A(t) \Psi[x H(t)]$, Duke Math. J. 32 (1965), 643-651. MR 32 \# 2638.

3. E. D. Rainville, Special functions, Macmillan, New York, 1960. MR 21 \#6447.

Department of Mathematics, University of Cincinnati, Cincinnati, OHio 45221

Faculté des Mathématique, Université de MontPellier, Montpellier, France 